\title{
Toward Understanding the Dynamics of Bidder Behavior in Continuous Combinatorial Auctions: Agent-Based Simulation Approach
}

\author{
Ali Mahdavi Adeli \\ Carlson School of Management \\ University of Minnesota \\ mahd0008@umn.edu
}

\author{
Gediminas Adomavicius \\ Carlson School of Management \\ University of Minnesota \\ gedas@umn.edu
}

\author{
Alok Gupta \\ Carlson School of Management \\ University of Minnesota \\ gupta037@umn.edu
}

\begin{abstract}
Combinatorial auctions represent sophisticated market mechanisms that are becoming increasingly important in various business applications due to their ability to improve economic efficiency and auction revenue, especially in settings where participants tend to exhibit more complex user preferences and valuations. While recent studies on such auctions have found heterogeneity in bidder behavior and its varying effect on auction outcomes, the area of bidder behavior and its impact on economic outcomes in combinatorial auctions is still largely underexplored. One of the main reasons is that it is nearly impossible to control for the type of bidder behavior in real world or experimental auction setups. We propose an agent-based modeling approach to replicate human bidder behavior in continuous combinatorial auctions and leverage our agents to simulate a wide variety of competition types, including experimentally unobserved ones that could not otherwise be studied. The capabilities of the proposed approach enable more comprehensive studies (via richer controlled experiments) of bidding behavior in the complex and highly dynamic decision environment of continuous combinatorial auctions.
\end{abstract}

\section{Introduction}

Combinatorial auctions [1] are important market mechanisms that allow bidders to bid on individual items as well as their combinations (bundles), which can lead to more efficient allocation of resources in complex market environments. While there has been research on a number of topics in this area - e.g., winner determination in combinatorial auctions, combinatorial auction designs, practicality of these designs for online marketplaces, and comparison of different auction mechanisms - the important issues related to bidder behavior in these auctions have been largely underexplored $[2,3]$. The main difficulty is that it is not possible to control for bidder behavior in experimental studies, which makes it hard to address a number of important and interesting research questions, for example, understanding how bidder behavior changes when facing different types of competition, and how these changes affect auction outcomes. In this paper, we use a data-driven approach to design and develop software agents that replicate human behavior in this complex trading mechanism. Our approach draws upon, but differs from, existing research on automated bidding agents [4-6] in that our agents are intended to replicate human bidding behavior, not to outperform human participants, compete against other agents, or optimize a given task. We leverage the agent-based modeling [711] approach to examine the effect of different bidder compositions (i.e., interaction of bidders with different bidding strategies) on auction outcomes and bidder behavior, using the case of continuous combinatorial auctions. Findings from our computational agent-based simulations allow for bottom-up theorizing [12] on the interaction of bidder behaviors and emerging auction outcomes.

\section{Background: continuous combinatorial auctions}

In combinatorial auctions bidders can bid on a single item or a bundle. At any time in the auction, any bid that has been submitted by an auction participant can be in one of three states: (a) winning, (b) dead, i.e., no chance of winning in the future, or (c) live, i.e., not currently dead or winning but may change to one these states depending on future bids. This is substantially different from single-item auctions where a bid can only be either winning or dead. Bids on non-overlapping bundles that create the highest revenue are winning bids, which are recalculated upon any new incoming bid. Given the three possible states of a bid, there are naturally two important bidding levels for any bundle $b$ at any given time in an auction: deadness level (DL) and winning level (WL), where $D L(b) \leq W L(b)$. A bid amount above the WL will make a bid winning, below the DL will result in a dead bid, and a bid amount in-between DL and WL will result in a live bid. Auction revenue 


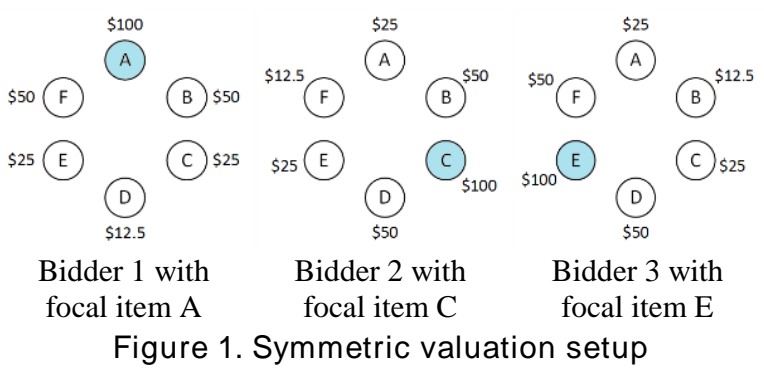

(or auctioneer revenue) is the amount obtained by the auctioneer via the winning bids, which equals the sum of winning bids' amounts in a first-price auction mechanism. Another important auction outcome is allocative efficiency, which measures how optimally items are distributed at the end of an auction and is defined as: Total Valuation of Auction Winners

Higher allocative efficiency is often stated as a desirable goal in auction literature, because it leads to greater social welfare [1]. The reason for this is that allocative efficiency is maximized when items are acquired by bidders who value them the most.

In this paper we use the following experimental continuous combinatorial auction (CCA) setup first introduced by Adomavicius and Gupta [13] as well as the dataset collected in their study [14], referred to as the "baseline experimental data" in the rest of the paper, to illustrate the proposed agent-based approach. In this setup, three bidders compete to acquire six items, representing six real-estate properties around a lake. A systematic valuation scheme is used where each bidder is designated a focal item, which has the highest value for that bidder among all items. The remaining items' value decreases by $50 \%$ the farther they are from the focal item (Figure 1). Complementarities are defined among items by adding a super-additive valuation of $10 \%$ for each adjoining item in a bundle. For example, if a bundle consists of focal item A (\$100 value), its neighbor item B (\$50 value), and B's neighbor C (\$25 value), then the valuation for bundle "ABC" is $(\$ 100+$ $\$ 50+\$ 25) \times(1+0.1+0.1)=\$ 210$, since there are two adjoining items in this bundle. Bidders are provided comprehensive information feedback throughout the auction, i.e., they can see bids placed so far in the auction, the provisional winning allocation at the current auction state, and the WL and DL for any bundle of interest. Since there are six items in each auction there are 63 possible bundles, i.e., all possible subsets of 6 items except for the empty set.

Using cluster analysis of bids and clicks generated by bidders, three stable bidder strategies/types have been identified in prior work [14], namely: Analyzers (A), Participators (P), and Explorers (E). These bidder types were shown to exhibit different behaviors in terms of several bidder-specific outcome variables (see Table 1 for cluster means and standard deviations):

- Bids: the number of bids placed by a bidder throughout the auction;

- Spans: the number of distinct bundles a bidder bids on throughout the auction;

- Surplus: the difference between a bidder's valuation and his/her winning bids upon auction end, i.e., a bidder's valuation for the won bundles minus the amount s/he has to pay for them;

- Effort: average number of clicks per bid during an auction, representing the level of information seeking effort by the bidder prior to submitting a bid (e.g., in terms of looking at DLs and WLs of various potential bundles of interest).

Analyzers (A) are the most rational bidders who spend more effort on analyzing the auction progress. Compared to the other two bidder types, a typical Analyzer places fewer bids on a smaller set of bundles for which s/he has higher valuation, and derives higher surplus as a result. Participators (P) do not spend much effort investigating the auction progress and maintain a participatory behavior. A typical Participator places more bids than Analyzers on a wider variety of bundles and derives a lower surplus than Analyzers, but higher surplus than Explorers. Explorers (E) spent the least effort on analyzing the auction environment and can display very random behavior. Compared to the other two types, a typical Explorer places the most bids on wider variety of bundles and derives the lowest surplus.

Since three bidders can participate in each auction and there are three different bidder types (A, P, and E), there can be 10 different possible bidder type combinations in a simple auction; we refer to these combinations of competing bidders as competition types. Only 3 out of these 10 possible competition types had enough observations in the baseline experimental CCAs to be included in statistical analysis.

\section{Modeling canonical bidder behaviors}

In a typical CCA, at any time in the auction a bidder can select a bundle s/he is interested in, check the DL and WL for the selected bundle, and decide to either place a bid of a certain monetary amount on the selected bundle or to not bid at this time. By analyzing the experimental bid-level and clickstream-level data, we observe different temporal bidding patterns for the three bidder types and characterize these behaviors in terms of "how often they bid at any auction state" (bidding frequency), "what they bid on" (bundle selection), and "how much they bid on a selected bundle" (bid amount). Other possible criteria were examined as well, 
Table 1. Mean and standard deviation for bidder specific variables in baseline experimental data

\begin{tabular}{|c|c|cc|cc|cc|cr|}
\hline Bidder Type & Number of Instances & \multicolumn{2}{|c|}{ Bids } & \multicolumn{2}{c|}{ Spans } & \multicolumn{2}{c|}{ Surplus } & \multicolumn{2}{c|}{ Effort } \\
\hline Analyzer & 4 & 15.50 & $(6.61)$ & 8.00 & $(3.56)$ & 77.50 & $(50.08)$ & 32.97 & $(26.20)$ \\
\hline Participator & 34 & 23.06 & $(8.84)$ & 9.88 & $(3.67)$ & 47.26 & $(44.65)$ & 17.45 & $(8.61)$ \\
\hline Explorer & 7 & 52.86 & $(16.88)$ & 21.57 & $(4.89)$ & 31.79 & $(28.45)$ & 11.15 & $(3.86)$ \\
\hline
\end{tabular}

but were ruled out for the purposes of this study, since they were either explainable in terms of the three above criteria or did not add significant value for our modeling purposes in terms of explaining the observed behavior. For example, bidders' "effort" is not explicitly modeled as a factor because the number of clicks indicates effort that has been exerted by a human bidder in order to explore the bidding environment before placing a bid, whereas the bidding environment is straightforwardly available to software agents. Or, "how quickly" a bid is placed is not included in the modelling of bidding agents because the overall bidding patterns are already captured by the bidding frequency parameter. This is advantageous, because it allows to straightforwardly scale the bidding dynamics to any desired auction duration. This feature is especially important for auction experiments that involve human participants as some of the bidders. For pure simulation-based experiments reported in this paper (i.e., where all auction participants are software agents), the actual "real-world" auction duration is not as meaningful. It is important to note that we model for the three distinct behaviors and the differences in temporal bidding patterns, not merely the bidder-specific outcome variables (i.e., Bids, Spans, and Surplus) that were used to identify, a posteriori, different bidding strategies. The bidding agents are expected to generate comparable bidder-specific outcomes, which will be verified as part of our validation.

To model each bidder type (Analyzer, Participator, and Explorer) in terms of the above behavioral aspects, we aggregate bidding data for bidders of the same type across all auctions. Since bidder behavior is likely

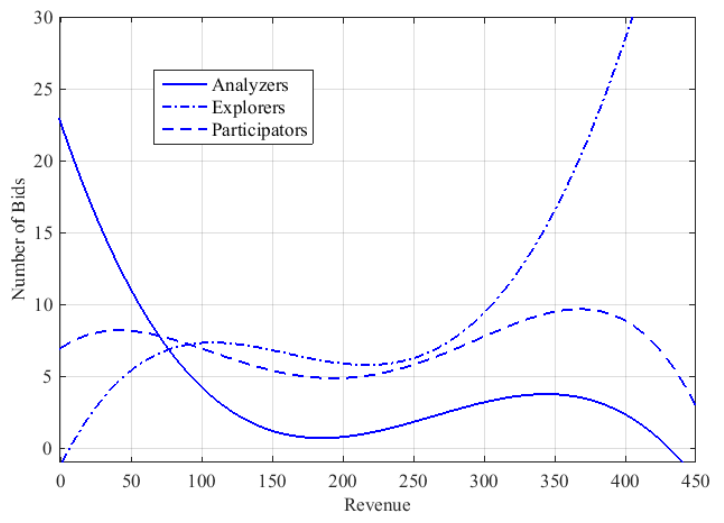

Figure 2. Comparing average bidding frequency functions for different bidder types dependent on the current state of the auction, we want to parameterize auction progress. Time is only one possible indicator of an auction state (e.g., represented as early, mid, and late portions of the auction), but it has certain limitations. A point of time is not a consistent indicator of auction progress (or auction state) across different auctions. This is because the duration of auctions varies significantly and mere passing of time does not always translate into bidder activity; e.g., bidders may not place any bids for some period of time during the auction. We propose to use auction revenue as a proxy for time and discretize the temporal bidding data based on revenue (revenue is increasing with time in any auction). Auction revenue is a time-invariant parameter that uniquely indicates auction state in the CCA context. Note that information about the DL and WL of any bundle is updated upon any new bid, and is available from the auction framework to any bidder who knows the current revenue.

\subsection{Bidding frequency}

To derive a dataset from which we can extract the dynamic bidding frequency aspect, we use the following steps to discretize and aggregate the experimental baseline data based on revenue:

- Define a revenue binwidth parameter and specify a width, e.g., binwidth $=\$ 25$.

- For each bidder, calculate the number of bids s/he has placed within each bin's revenue range; this is the bidder's bidding frequency at each revenue bin. For example, if a bidder has placed four bids when auction revenue is between $\$ 25$ and $\$ 50$ (i.e., within the $2^{\text {nd }}$ bin's revenue range when using binwidth $=\$ 25$ ), his bidding frequency equals 4 for the $2^{\text {nd }}$ bin.

- For each bidder type (A, P, and E), calculate the average bidding frequency and standard deviation at each bin by aggregating the bidding frequency values of all same-type bidders for that bin. E.g., if three Analyzers have the bidding frequency values of 4,5 , and 6 for the $2^{\text {nd }}$ bin, the average bidding frequency value for Analyzers at the $2^{\text {nd }}$ bin equals 5, with a (bidding frequency) standard deviation of 1 .

The result is average bidding frequency and standard deviation series for each bidder type, which represent how bidding frequency changes with auction state. To model these three distinct bidding frequency patterns, we fit a function on these series. For each bidder type, 
the function fitted on the average bidding frequency series models the bidding frequency of a typical bidder of that type. The function fitted on the standard deviation series models the difference among bidders of the same type. Since binwidth choice affects the resulting series and fitted functions, the above procedure is repeated for several different binwidth values $(\$ 10$, $\$ 15 \ldots$... \$40) and various fitting functions. The most suitable parameterization is derived for each bidder type based on goodness of fit and the expert-based understanding of bidder behavior. The resulting functions for the three bidder types are plotted in Figure 2. A linear fit is used for all standard deviation functions, which represents a rough one standard deviation confidence interval around the mean (the confidence intervals are not shown in Figure 2).

\subsection{Bundle selection}

Bidders' interest in different bundles depends on their valuation for the items, which does not change with auction state (is static). However, given the same valuation scheme and identical focal items, the three bidder types vary in their propensity to bid on different bundles. For example, Analyzers are much more likely to bid on a bundle that consists of all six items compared to Participators and Explorers. To model the bundle selection aspect, we use the aggregated bidding data across all auctions for each bidder type and derive discrete probability distributions that specify the likelihood of a bundle being selected. We derive a probability mass function for each bidder type. A bidding agent determines which bundle to bid on by making a random pick from its bundle selection distribution given its focal item.

\subsection{Bid amount}

To model how much different bidder types bid on bundles across different auctions, we need to take care of the changing WL and DL of bundles as the auctions proceed. We introduce a bid-level variable that represents the amount bid on a bundle relative to the bundle's WL and DL, and calculate it for every bid as following:

- If WL = DL: bid-level= bid-amount / DL;

- If WL > DL: bid-level=(bid-amount - DL)/(WL - DL);

- If WL and/or DL are not yet set for the bundle (i.e., in the beginning of the auction), the initial bid-amounts are used to establish separate starting-bid distributions, which are used to determine an amount for the first bids placed by bidding agents.

This bid-level variable allows us to meaningfully analyze the bid-amount aspect of the three bidder types

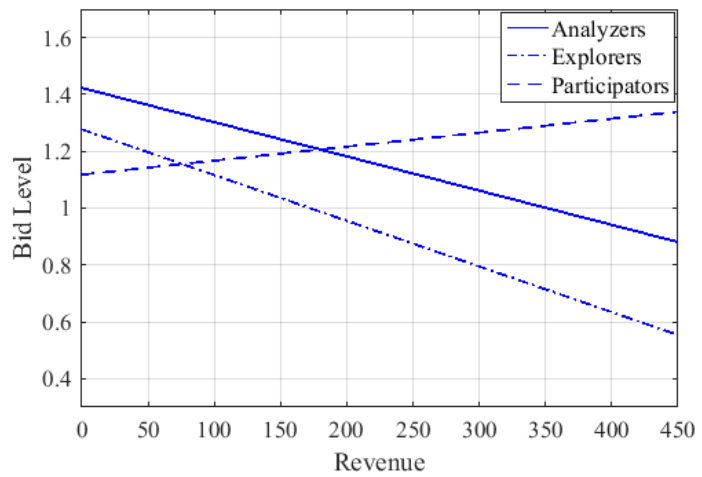

Figure 3. Comparing average bid-level functions

across different auctions and across different auction states. We discretize and aggregate the bid-levels we have derived for each bidder type (using steps similar to what we explained for bidding frequency) and derive average bid-level and standard deviation series that represent how bid-level changes with auction state. To model the distinct patterns for the three different bidder types, we repeat the process using several revenue binwidths to derive the series, use various fitting functions, and select the best fit (the approach is similar to what we did for bidding frequency). The difference between the resulting functions for the three bidder types are shown in Figure 3. To model the difference among bidders of the same type in terms of bid-level, we use a linear fit on the standard deviation series.

A bidding agent determines the amount it bids on a selected bundle by specifying a bid-level and knowing the WL and DL for the bundle at any given auction state.

\subsection{Agent-based auction simulations}

Each of the three main aspects of bidder behavior (bidding frequency, bid amount, and bundle selection) are implemented in our agents as separate modules. The fourth, bid-decision module determines the agent's final decision of whether or not to bid, after combining values returned by the three above modules and considering the agent's bidding history so far. When an auction simulation starts, each agent determines the expected number of bids it is going to place at the current auction state via its bidding frequency module. Each time an agent intends to bid, it selects a specific bundle and a corresponding bid-amount, as determined by its Bundle Selection and Bid Amount modules. Based on the agent's valuation for the selected bundle, the intended bid amount, and the bids placed so far, it decides whether or not to place the current bid using its biddecision module. In experimental CCAs, the auction ends when none of the bidders places a bid for an extended period of time (e.g., 5 minutes). In the simulation environment, we model this soft stopping 
rule by defining a non-activity parameter. When an agent decides to not place the bid the non-activity count is increased by one, which models bidders' inactivity in experimental CCAs as implied by effort that does not lead to placing a bid. When the aggregate non-activity count reaches a certain threshold the auction simulation stops. The value for this stopping threshold models the extended inactivity time that ends real auctions.

\section{Data-driven validation of agent-based simulations}

In designing our bidding agents, we postulated that three main aspects (namely, bidding frequency, bundle selection, and bid amount) characterize different bidding behaviors. In this section we verify whether our bidding agents, built based on the aforementioned assumptions, correctly replicate human bidders' behavior under similar conditions (i.e., under the same competition types) by statistically comparing outcome variables generated by our simulation model with outcome variables from experimental CCAs. We assess the validity of our agent-based simulations in terms of matched bidder-specific variables (Bids, Spans, and Surplus) and auction outcome (revenue), by replicating competition types observed in experimental CCAs, namely APP, EPP, and PPP. Our approach follows the recommended practices for verification and validation of simulation models by leveraging the notions and frameworks of model output behavior, point validity, distributional validity, and pattern validity [7, 15-17].

\subsection{Replicating bundle diversity and Bids}

Figures 4 and 5 show the side-by-side comparison of Bids (number of bids) and Spans (diversity of bundles) variables between human bidders and bidding agents, respectively. The horizontal axis indicates the human bidder or bidding agent type (e.g., "E Agent" stands for

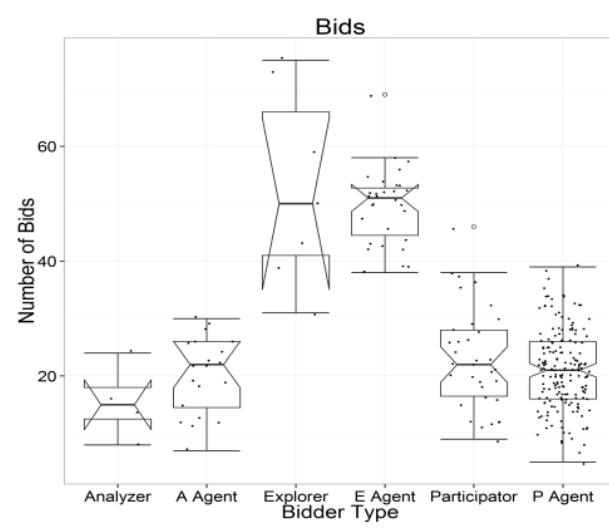

Figure 4. Comparing Bids variable between human bidders and bidding agents

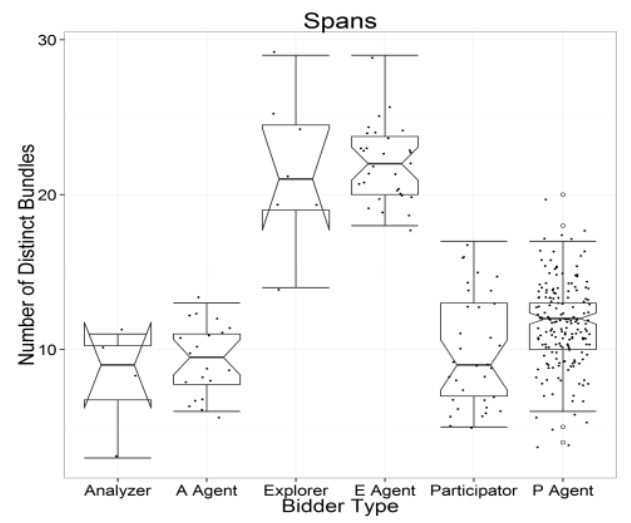

Figure 5. Comparing Spans variable between human bidders and bidding agents

Explorer type bidding agents). We use t-tests, Wilcoxon rank-sum (WRS aka. Mann-Whitney U) tests, and Kolmogorov-Smirnov (KS) tests to compare the data generated by our bidding agents in simulated auctions with data from experimental auctions generated by human bidders. When t-tests and WRS tests indicate no significant difference, this provides evidence with respect to point validity; i.e., the means and medians of

Table 2. Comparing bidder specific variables between human bidders and bidding agents

\begin{tabular}{|c|c|c|c|c|c|c|c|c|}
\hline \multicolumn{3}{|c|}{$\begin{array}{l}\text { H0: Human }=\text { Agent } \\
\text { H: Human } \neq \text { Agent }\end{array}$} & \multicolumn{2}{|c|}{ Wilcox Rank Sum } & \multicolumn{2}{|c|}{ t-test } & \multicolumn{2}{|c|}{$\begin{array}{l}\text { Kolmogorov } \\
\text { Smirnov }\end{array}$} \\
\hline & \multicolumn{2}{|c|}{$\begin{array}{c}\text { Comparing Human vs. Bidding Agent } \\
\text { of Type }\end{array}$} & W & p-value & $\mathrm{T}$ & p-value & $\mathrm{D}$ & p-value \\
\hline \multirow{3}{*}{ Bids } & \multicolumn{2}{|l|}{ Analyzer } & 25.5 & 0.277 & -1.296 & 0.26 & 0.45 & 0.5095 \\
\hline & \multicolumn{2}{|l|}{ Explorer } & 109.5 & 0.876 & 0.49 & 0.6394 & 0.395 & 0.3379 \\
\hline & \multicolumn{2}{|l|}{ Participator } & 3325 & 0.2782 & 1.266 & 0.2128 & 0.125 & 0.7635 \\
\hline \multirow{6}{*}{ Spans } & \multirow{3}{*}{\multicolumn{2}{|c|}{$\begin{array}{c}\text { Analyzer } \\
\text { Explorer } \\
\text { Participator }\end{array}$}} & 31.5 & 0.532 & -0.73 & 0.5113 & 0.25 & 0.9853 \\
\hline & & & 98.5 & 0.814 & -0.243 & 0.815 & 0.295 & 0.7056 \\
\hline & & & $2096.5^{* *}$ & 0.0062 & $-2.499^{*}$ & 0.0166 & $0.342^{* *}$ & 0.0026 \\
\hline & \multirow{3}{*}{$\begin{array}{l}\text { Participators broken down by } \\
\text { competition type }\end{array}$} & $\mathrm{P}$ in $\mathrm{APP}$ & 123.5 & 0.3146 & -1.517 & 0.1703 & $0.5^{+}$ & 0.0713 \\
\hline & & $\mathrm{P}$ in $\mathrm{EPP}$ & 269.5 & 0.3368 & -0.832 & 0.4197 & 0.212 & 0.797 \\
\hline & & $\mathrm{P}$ in $\mathrm{PPP}$ & $348.5^{*}$ & 0.0198 & $-1.814^{+}$ & 0.087 & $0.387^{*}$ & 0.0476 \\
\hline
\end{tabular}

Significance levels: $* * * 0.001, * * 0.01, * 0.05,+0.1$ 
the compared variables are not significantly different. We also run two-sample KS tests to verify whether variables generated by humans and bidding agents have the same distribution. When the KS test does not show a significant difference, this provides evidence with respect to distributional validity for the compared variable. Table 2 shows the comparison of Bids and Spans variables between human bidders and bidding agents of the same type. Validation in terms of Surplus is excluded to save space; the simulation model demonstrates pattern validity in terms of Surplus.

Based on these results, all three bidding agent types generate comparable number of bids (i.e., captured by Bids variable) as human bidders. Since none of the tests show a significant difference in Bids for any of the three bidder types, our simulation model has distributional validity in terms of Bids. The bundle diversity (i.e., Spans variable) generated by Analyzer and Explorer type bidding agents has the same distribution (as well as the same mean and median) as Spans generated by human bidders. Participator type bidding agents seem to slightly differ from human bidders; i.e., Spans difference is significant at the $1 \%$ level (p-values close to 0.01 for all three tests). However, by looking at Participator data broken down by competition, we see that the difference in Spans is only marginally significant when Participators compete against other Participators (i.e., in auctions with PPP type competition); there is no significant difference in Spans between Participator type human bidders and bidding agents in auctions with EPP and APP competitions. Nevertheless, the relative difference in bundle diversity among different bidding agent types always matches those generated by human bidders.

\subsection{Replicating auction outcomes}

We compare auction revenue between simulated and experimental auctions with identical competition types to verify the similarity of auction-specific outcomes. We use WRS tests to compare revenue between auctions with different competition types in Table 3 . The results

Table 3. Comparing auction revenue across competitions observed in experimental auctions

\begin{tabular}{|c|c|c|c|c|c|}
\hline & \multicolumn{5}{|c|}{ Revenue in Competition of Type: } \\
\hline $\begin{array}{c}\text { Experimental } \\
\text { Auctions }\end{array}$ & APP & & EPP & $<*$ & PPP \\
\hline Mean (stdev) & $258(141)$ & & $333.7(66.6)$ & & $381.6(49.9)$ \\
\hline $\begin{array}{c}\text { Auction } \\
\text { Simulations }\end{array}$ & APP & & EPP & $<* * *$ & PPP \\
\hline Mean (stdev) & $468(22.8)$ & & $463.4(21.7)$ & & $482(22.1)$ \\
\hline
\end{tabular}

Significance levels: $* * * 0.001, * * 0.01, * 0.05,+0.1$,

$\approx$ no significant difference show that auction revenue has a similar pattern in both experimental and simulated auctions (i.e., APP $\approx \mathrm{EPP}<$ $\mathrm{PPP}$ ), even though average revenue is different between experimental and simulated auctions with the same type of competition. This difference is because our bidding agents do not stop bidding pre-maturely as is the case in a few experimental auctions with APP and EPP competition type, as well as, the small sample size in experimental auctions (as implied by the very large standard deviation for APP and EPP in Table 3).

In summary, the validation shows that our agentbased simulation model has at least pattern validity when we assess our model in terms of bidder specific outcome variables (i.e., Bids, Spans, and Surplus) and auction outcome (i.e., revenue). Pattern validity is sufficient to leverage our model for studying dynamics of bidder behavior and consequent auction outcomes using agent-based simulations.

\section{Leveraging bidding agents}

Our agent-based auction simulations allow us to explore the effect of competition on the dynamics of bidder behavior and auction outcomes. Note that agents are not explicitly programmed to behave differently based on the competition they face. Instead, we are interested in finding out whether the bidding dynamics that result from different competitions, while the bidder agents bid based on the coded canonical behaviors, provide interesting insights into potential auction outcomes through "emergent" behaviors of the agents under different competitive environments. We run 100 auction simulations for each of the 10 possible competition types (total of 1000 auction simulations) for this part of the study, using the same valuation setup used in experimental CCAs. Even though the simulation platform allows us to run auctions with more than 3 bidding agents (e.g., 6 bidders), in this study we have focused on running simulations with 3 bidding agents in each auction so that the results can be compared with results from experimental auctions.

\subsection{Effect of competition on bidder behavior}

Each bidder can face 6 types of competition based on the composition of bidding strategies it encounters in the auction, namely: AA (competing against two Analyzers), AP (competing against an Analyzer and a Participator), AE, PP, EP, and EE. Even if we assume that bidders' behavior is endowed, we can clearly hypothesize that the type of competition a bidder faces affects his/her behavior, including both the number of placed bids (the Bids variable) and the diversity of bundles bid on (the Spans variable). We use ANOVA 
Table 4. ANOVA of factors influencing bidding agents' behavior and surplus

\begin{tabular}{|c|c|c|c|}
\hline & Bids & Spans & Surplus \\
\cline { 2 - 4 } & $\mathrm{F}$ & $\mathrm{F}$ & $\mathrm{F}$ \\
\hline Bidder Type & $816.01 * * *$ & $1058.4 * * *$ & $46.1 * * *$ \\
\hline Comp. Type & $54.4 * * *$ & $13.3 * * *$ & $11.1 * * *$ \\
\hline $\begin{array}{c}\text { Bidder Type } \times \\
\text { Comp. Type }\end{array}$ & $13.3 * * *$ & $14.8 * * *$ & $5.4 * * *$ \\
\hline $\mathrm{R}^{2}$ & 0.7742 & 0.7956 & 0.1867 \\
\hline Adj. $\mathrm{R}^{2}$ & 0.7729 & 0.7944 & 0.1821 \\
\hline & $\begin{array}{c}\mathrm{F}(17,2982) \\
=601.5 * * *\end{array}$ & $\begin{array}{c}\mathrm{F}(17,2982) \\
=682.6 * * *\end{array}$ & $\begin{array}{c}\mathrm{F}(17,2982) \\
=40.28 * * *\end{array}$ \\
\hline
\end{tabular}

Significance levels: $* * * 0.001, * * 0.01, * 0.05,+0.1$

(see Table 4) to study how bidders' own type, the competition type they face, and the possible interaction of these two factors affect each of these bidder-specific outcome variables (Bids, Spans, and Surplus).

The models are highly significant ( $\mathrm{p}$-value $\leq 0.001$ ) with a high explanation of variance (adjusted $\mathrm{R}^{2}>75 \%$ ). This tells us that bidding agents' own strategy and the competition type they face both significantly affect the number of bids, the diversity of bundles they bid on, and their economic welfare (as measured by surplus). The significant interactions indicate that the effect of competition type on agent behavior depends upon the agent's bidding strategy. We use interaction plots to uncover the patterns of these interactions in Figures 6 and 7. Each point shows the group mean for a certain agent type facing a specific competition (e.g., Analyzers facing AP competition), and the error bars indicate $95 \%$ confidence intervals. These plots provide a guideline for further post-hoc tests to verify significant differences.

The general differences among the three bidding agent types (Participator, Analyzer, and Explorer) in Figure 6 are consistent with the differences among the three bidding strategies observed in experimental CCAs (Figures 4 and 5). To study these patterns, we use pairwise t-tests and WRS tests (when we suspect nonnormal distributions) to compare Bids and Spans

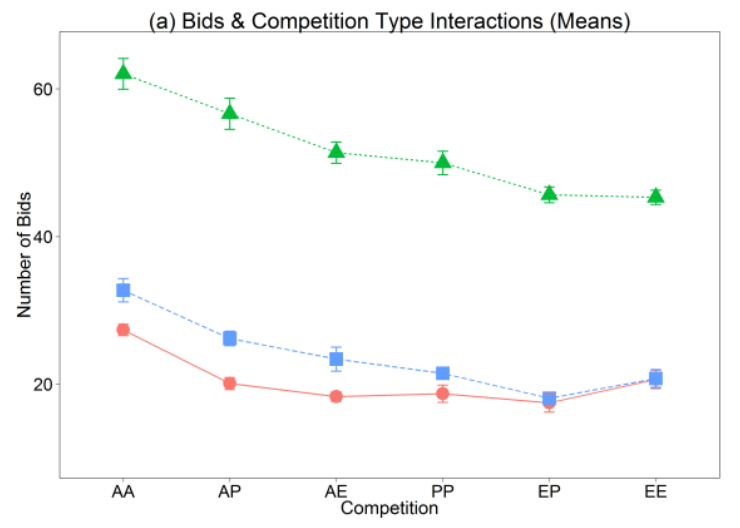

between any two types of competition, for each bidder type. Tables 5, 6, and 7 report the mean values for these variables under different competition types as well as significant differences for each of the three bidding agent types (when the t-test and WRS test results have different significance levels, the less significant test is shown). Figure 7 shows that Analyzers generally derive the highest surplus and Explorers derive the lowest surplus, irrespective of the type of competition they face.

For Explorers, as the competition changes from AA to AP to PP, there is a significant and consistent drop in both the overall number of bids they place (Bids), and the overall variety of bundles they bid on (Spans); see Table 5. Interestingly, Explorers surplus drops and becomes more negative (Figure 7) while we initially expected Surplus to drop with Bids and Spans. This implies that a single Explorer benefits from tougher competition (i.e., AA) by losing less. Once there are two Explorers in the competition, the third bidder's strategy doesn't significantly affect Explorers' bidding behavior in terms of Bids and Spans (EP vs EE competition types) unless the third bidder is an Analyzer (AE competition type), which makes for a slightly tougher competition where Explorers place slightly more bids on more various bundles.

For Participators', competing against two Analyzers is the toughest they have the highest Bids and Spans (Table 6), while having the lowest surplus, under this competition (Figure 7). Competition intensity reduces once one of the Analyzers is replaced by a Participator. Bidding against PP or AE competition types make for a moderate competition, based on Participators' Bids and Spans, but they make more surplus under the AE competition. Participators place the least number of bids on the fewest number of distinct bundles when they bid against EP competition type, while deriving higher surplus, which implies a weak competition. Interestingly, bidding against two

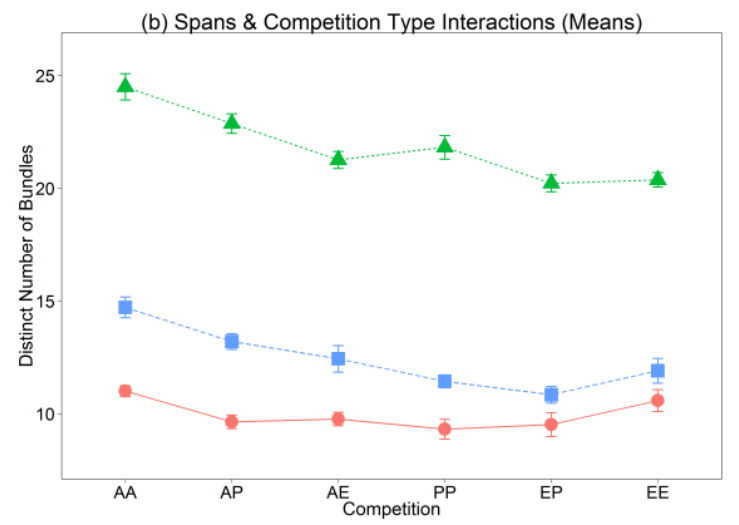

Bidding Agent Type Analyzer $A$ Explorer $=$ Participator Figure 6. Effect of competition on bidding agent behavior under different bidding strategies 


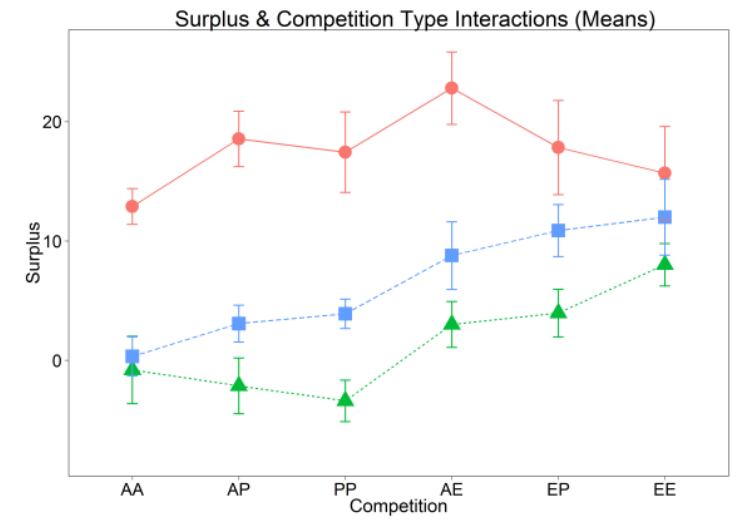

Bidding Agent Type Analyzer $A$ Explorer - Participator Figure 7. Effect of competition on bidder surplus

Explorers makes Participators place more bids on more various bundles, probably in response to the frequent random bids placed by Explorers on various bundles.

Analyzers are the most rational among the three bidder types who make the most effort to place smarter bids that maximize their surplus. Competing against two Analyzers is considered the toughest competition other bidders can face (including an Analyzer). The bidding agents' behavior supports this hypothesis, as indicated by the highest Bids and Spans for all three agent types when they face competition of type AA (see Figure 6). Analyzers' Bids and Spans both significantly decrease, while their surplus increases (Figure 7) when one of the Analyzers is substituted with a Participator. Analyzer type agents place less bids when facing AE, PP, or EP competitions compared to auctions with AP competition type, but the variety of bundles they bid on (Spans) does not significantly decrease (Table 7). Analyzers' derive the highest surplus when facing AE competition, where both Analyzers are exploiting the random behavior of a single Explorer. For Analyzers, EP competition is not significantly different from AE and PP competitions in terms of Bids and Spans, while it is the least intense for Explorers and Participators. Competing against two Explorers seems to confuse Analyzer agents as they behave similar to when they face AA and AP competition types, even though EE competition type is expected to be less demanding. Under EE competition, a Analyzer agents ends up placing more bids on more various bundles trying to make sense of, and respond to, the rather random behavior of Explorers and derive a highly uncertain surplus (as implied by Analyzers fattailed surplus distribution under EE competition: mean $=15.7$, median $=1$, skew $=0.94$, kurtosis $=-0.39$ ).

Our analysis of agents' emerging behaviors provides insights into human bidders' probable behaviors under similar competitions. Participation of Analyzers in an auction generally makes for a tougher competition. Other bidder types, including another Analyzer, place more bids (higher Bids) on a wider variety of bundles (higher Spans) in the presence of Analyzers. Explorers generally make for a weaker competition. Other bidder types, including another Explorer, generally bid less frequently on less various bundles when competing with Explorers. However, Explorers' rather unexpected behavior in terms of placing random bids on various bundles can also confuse other bidders and makes for more uncertain outcomes in terms of surplus. When the majority of bidders are Explorer types, other bidders (i.e., bidders facing EE competition type) can get confused if they overanalyze Explorers' random

Table 5. Comparing Explorer behavior across different competition types

\begin{tabular}{|c|c|c|c|c|c|c|c|c|c|c|c|c|}
\hline & & \multicolumn{11}{|c|}{ Explorer Facing Competition of Type: } \\
\hline \multirow{2}{*}{ Bids } & \multirow[b]{2}{*}{ Mean } & AA & $>* * *$ & AP & $>* * *$ & $\mathrm{PP}$ & $\approx$ & $\mathrm{AE}$ & $>* * *$ & EP & $\approx$ & $\mathrm{EE}$ \\
\hline & & 62.04 & & 56.64 & & 49.98 & & 51.37 & & 45.655 & & 45.303 \\
\hline \multirow{2}{*}{ Spans } & \multirow[b]{2}{*}{ Mean } & AA & $>* * *$ & AP & $>* * *$ & $\mathrm{PP}$ & $>^{+}$ & $\mathrm{AE}$ & $>* * *$ & EP & $\approx$ & $\mathrm{EE}$ \\
\hline & & 24.5 & & 22.87 & & 21.82 & & 21.26 & & 20.225 & & 20.377 \\
\hline
\end{tabular}

Significance levels: $* * * 0.001, * * 0.01, * 0.05,+0.1, \approx$ no significant difference

Table 6. Comparing Participator behavior across different competition types

\begin{tabular}{|c|c|c|c|c|c|c|c|c|c|c|c|c|}
\hline & & \multicolumn{11}{|c|}{ Participator Facing Competition of Type: } \\
\hline \multirow{2}{*}{ Bids } & \multirow[b]{2}{*}{ Mean } & AA & $>* * *$ & $\mathrm{AP}$ & $>* *$ & $\mathrm{AE}$ & $>*$ & $\mathrm{EE}$ & $\approx$ & PP & $>* * *$ & EP \\
\hline & & 32.72 & & 26.19 & & 23.39 & & 20.77 & & 21.457 & & 18.07 \\
\hline \multirow{2}{*}{ Spans } & \multirow[b]{2}{*}{ Mean } & $\mathrm{AA}$ & $>* * *$ & $\mathrm{AP}$ & $>*$ & $\mathrm{AE}$ & $\approx$ & $\mathrm{EE}$ & $\approx$ & $\mathrm{PP}$ & $>*$ & EP \\
\hline & & 14.72 & & 13.205 & & 12.44 & & 11.91 & & 11.44 & & 10.845 \\
\hline
\end{tabular}

Table 7. Comparing Analyzer behavior across different competition types

\begin{tabular}{|c|c|c|c|c|c|c|c|c|c|c|c|c|}
\hline & & \multicolumn{11}{|c|}{ Analyzer Facing Competition of Type: } \\
\hline \multirow{2}{*}{ Bids } & \multirow[b]{2}{*}{ Mean } & AA & $>* * *$ & $\mathrm{EE}$ & $\approx$ & AP & $>* * *$ & $\mathrm{AE}$ & $\approx$ & PP & $\approx$ & EP \\
\hline & & 27.35 & & 20.63 & & 20.09 & & 18.31 & & 18.71 & & 17.45 \\
\hline \multirow{2}{*}{ Spans } & \multirow[b]{2}{*}{ Mean } & $\mathrm{AA}$ & $>* *$ & $\mathrm{EE}$ & $>* *$ & AP & $\approx$ & $\mathrm{AE}$ & $>^{+}$ & PP & $\approx$ & EP \\
\hline & & 11.01 & & 10.59 & & 9.645 & & 9.77 & & 9.32 & & 9.52 \\
\hline
\end{tabular}


behavior and may place more bids on more diverse bundles as a result, even though the competition is not necessarily tougher. Analyzers, due to their more investigative nature, are more likely to be affected in this way than Participators. Participators are the middle ground in terms of bidding frequency and bundle diversity. They are less competitive than Analyzers but more competitive than Explorers, without Explorers' random bidding behavior. Their participatory behavior can neutralize the effect of Analyzers' competitiveness and Explorers' random behavior (e.g., in auctions with APE competition).

Based on our analysis, we characterize competition types as follows: AA - intense, AP - strong, PP consistent moderate, AE - uncertain moderate (or, moderate but uncertain), EP - weak, EE - confusing weak (or, weak but confusing); sorted by intensity and uncertainty in decreasing order we have: AA, AP, PP, $\mathrm{AE}, \mathrm{EP}$, and $\mathrm{EE}$.

\subsection{Effect of competition on auction outcomes}

Our results in Table 8 indicate that bidding agents' emergent behaviors under different competition types lead to significant differences in auction revenue and allocative efficiency. Table 9 shows the overall trend in auction revenue across all different competitions as well as results of pairwise comparisons using t-tests to verify significant differences in auction revenue; Table 10 shows these trends and comparisons for allocative efficiency. Auctions with PPP competition type, which produce the highest auction revenue, also make for consistently higher allocative efficiency, due to Participators' moderate behavior in absence of other bidder types. Based on these results, we can argue that Explorer agents' random behavior provides other bidder types a better opportunity to derive higher surplus. The auctioneer derives the lowest average revenue in auctions with EAA competition, where two Analyzer agents derive the highest surplus by exploiting the random behavior of a single Explorer agent. Under EEA competition type, there is only one Analyzer agent who exploits the randomness of Explorer agents, leaving the
Table 8. ANOVA for effect of competition types on auction outcomes in auction simulations

\begin{tabular}{|c|c|c|}
\hline & Auction Revenue & $\begin{array}{c}\text { Allocative } \\
\text { Efficiency }\end{array}$ \\
\cline { 2 - 3 } & $\mathrm{F}$ & $\mathrm{F}$ \\
\hline Competition Type & $168.37 * * *$ & $51.764 * * *$ \\
\hline $\mathrm{R}^{2}$ & 0.3363 & 0.1348 \\
\hline Adj. $\mathrm{R}^{2}$ & 0.3343 & 0.1322 \\
\hline & $\mathrm{F}(9,2990)=$ & $\mathrm{F}(9,2990)=$ \\
$168.4 * * *$ & $51.76 * * *$ \\
\hline
\end{tabular}

Significance levels: $* * * 0.001, * * 0.01, * 0.05,+0.1$

auctioneer with relatively higher revenue (as compared to auctions with EAA competition). Participator agents can also take advantage of opportunities created by Explorers (i.e., EPP and EEP competition types) but cannot exploit them like Analyzers do (i.e., EAA, EEA, and APE competition types). Analyzer agents not only exploit Explorers' random behavior but can also exploit opportunities created by Participators (i.e., APE, PAA and APP competition types). There is no significant difference in Participators' surplus when facing EP or EE competitions which leads to a similar revenue in auctions with EPP or EEP competition.

\section{Discussion \& Conclusion}

The key contributions of this paper are three-fold. First, we propose and design a data-driven approach for developing software agents that are able to replicate multi-faceted human bidder behavior in complex decision environments of CCAs, which are important, sophisticated market mechanisms that are becoming increasingly used in various business applications. Our study is the first to use an agent-based modeling approach for this purpose. The validity of our agents is demonstrated by replicating bidder specific variables and auction parameters that were observed in experimental CCAs with real users. Second, we successfully leverage these agents to better understand dynamics of bidder behavior and explore competition types not observed in experimental auctions. The simulations indicate how different competition types

Table 9. Comparing revenue across different competition types

\begin{tabular}{|c|c|c|c|c|c|c|c|c|c|c|c|c|c|c|c|c|c|c|}
\hline \multicolumn{19}{|c|}{ Revenue under Competition Type } \\
\hline EAA & $<* * *$ & EEA & $\approx$ & APE & $\approx$ & PAA & $<*$ & AAA & $<* * *$ & EEE & $<*$ & EPP & $\approx$ & APP & $\approx$ & EEP & $<* * *$ & PPP \\
\hline & & EEA & & $<*$ & & PAA & & & & & & EPP & & $\approx$ & & EEP & & \\
\hline
\end{tabular}

Significance levels: $* * * 0.001, * * 0.01, * 0.05,+0.1, \approx$ no significant difference

Table 10. Comparing allocative efficiency across different competition types

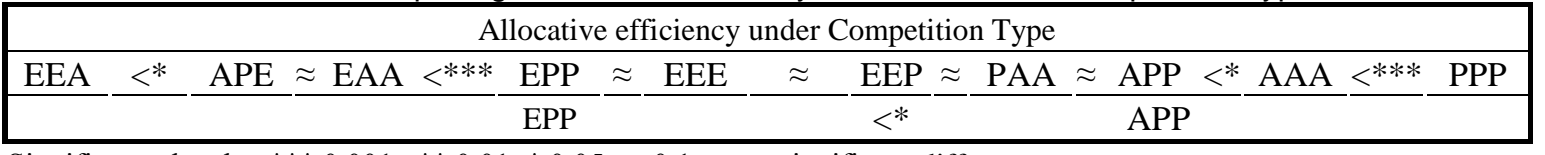

Significance levels: $* * * 0.001, * * 0.01, * 0.05,+0.1, \approx$ no significant difference 
affect auction outcomes, such as revenue, and show that different bidder types are affected differently by the type of competition they face in ways that are not always intuitive. We analyzed bidding agents' emergent behaviors under different competition types and explained the probable underlying mechanisms that lead to these different behaviors and welfare outcomes. The same arguments allowed us to consistently explain differences in auction outcomes, which further supports the consistency of our agent-based modeling approach in simulating human bidder behavior. We would expect actual bidders' behavior to change consistent with our agents' emergent behaviors under similar competitions. This understanding is necessary for possible customer segmentation and market designs to attract different participant types to certain auctions (designing incentive mechanisms). And third, the capabilities of our modeling approach allow us to design future experimental studies to analyze how human bidder behavior and auction outcomes are affected under different competition scenarios. A unique outcome of this work is that it enables controlled experiments where human participants compete with software agents that exhibit bidding strategies of our choice, which allows us to address interesting questions that could not otherwise be answered. For example, whether bidders' behavior is indeed endowed or learned? And if bidders can learn, what are the most effective learning paths to encourage certain types of behavior? Our proposed approach provides unique opportunities to further our understanding of bidder behavior in a complex, competitive, and dynamic decision environment.

Our study has implications for the design and implementation of combinatorial auctions in digital marketplaces. We show how heterogeneity in auction outcomes is (partly) driven by competition; for example, certain competition types (i.e., composition of Analyzer and Explorer type behaviors) lead to lower auctioneer revenue as well as suboptimal allocative efficiency (i.e., low on average, with high variability). Auctioneers can utilize this understanding to incentivize participants to adopt different strategies (e.g., changing exploratory behavior to participatory behavior) that would benefit bidders as well as auctioneers. Moreover, participants' experience in those auctions in terms of surplus and/or the possible frustration from having a bad experience (e.g., spending a lot of effort without winning the desired items or unexpectedly deriving lower surplus), as a result of the competition they face, can affect their future decision of whether or not to participate in such markets (e.g., affecting sustainability these markets). Our findings can be utilized to design market mechanisms that would discourage or prevent exploratory behavior that leads to undesired types of competition. Lastly, we also contribute to the design of more user-centric artificial bidding agents by developing software agents that demonstrate strategic and human-like behavior in a complex market environment.

\section{References}

[1] P. Cramton, et al., "Combinatorial auctions," 2006.

[2] T. Scheffel, et al., "An experimental comparison of linear and nonlinear price combinatorial auctions," Information Systems Research, vol. 22, pp. 346-368, 2011.

[3] Y.-H. Park and E. T. Bradlow, "An integrated model for bidding behavior in Internet auctions: Whether, who, when, and how much," Journal of Marketing Research, vol. 42, pp. 470-482, 2005.

[4] M. He, et al., "On agent-mediated electronic commerce," IEEE Transactions on knowledge and data engineering, vol. 15, pp. 985-1003, 2003.

[5] J. Collins, et al., "Pushing the limits of rational agents: the trading agent competition for supply chain management," $A I$ magazine, vol. 31, p. 63, 2010.

[6] M. P. Wellman, et al., Autonomous bidding agents: Strategies and lessons from the trading agent competition: Mit Press, 2007.

[7] N. Gilbert, Agent-based models: Sage, 2008.

[8] C. M. Macal and M. J. North, "Tutorial on agent-based modeling and simulation," in Proceedings of the 37th conference on Winter simulation, 2005, pp. 2-15.

[9] E. Bonabeau, "Agent-based modeling: Methods and techniques for simulating human systems," Proceedings of the National Academy of Sciences, vol. 99, pp. 7280-7287, 2002.

[10] N. R. Jennings, et al., "A roadmap of agent research and development," Autonomous agents and multi-agent systems, vol. 1, pp. 7-38, 1998.

[11] N. R. Jennings and M. Wooldridge, "Applications of intelligent agents," in Agent technology, ed: Springer, 1998, pp. 3-28.

[12] J. H. Miller and S. E. Page, Complex adaptive systems: An introduction to computational models of social life: Princeton university press, 2009.

[13] G. Adomavicius and A. Gupta, "Toward comprehensive real-time bidder support in iterative combinatorial auctions," Information Systems Research, vol. 16, p. 169, 2005.

[14] G. Adomavicius, et al., "Effect of information feedback on bidder behavior in continuous combinatorial auctions," Management Science, vol. 58, pp. 811-830, 2012.

[15] R. G. Sargent, "Verification and validation of simulation models," in Proceedings of the 37th conference on Winter simulation, 2005, pp. 130-143.

[16] R. L. Van Horn, "Validation of simulation results," Management Science, vol. 17, pp. 247-258, 1971.

[17] G. B. Kleindorfer, et al., "Validation in simulation: various positions in the philosophy of science," Management Science, vol. 44, pp. 1087-1099, 1998. 